\title{
Infectomic Insights into the Roles of Exosymbiosis-Endosymbiosis Imbalance (EESI) in HIV-1 and SIV Infections
}

\author{
Sheng-He Huang ${ }^{1-3^{*}}$ and Yan-Hong Zhou ${ }^{1^{*}}$ \\ ${ }^{1}$ Hubei Bioinformatics and Molecular Imaging Key Laboratory, College of Life Science and Technology, Huazhong University of Science and Technology, Wuhan, China \\ ${ }^{2}$ Department of Paediatrics, Saban Research Institute of Children's Hospital Los Angeles, University of Southern California, Los Angeles, California, United states \\ ${ }^{3}$ Department of Microbiology, School of Publics Health and Tropical Medicine, Southern Medical University, Gaungzhou, China \\ ${ }^{*}$ Corresponding authors: Yan-Hong Zhou, Hubei Bioinformatics and Molecular Imaging Key Laboratory, College of Life Science and Technology, Huazhong University \\ of Science and Technology, Wuhan, China, Tel: 213-440-2528; E-mail: shhuang@usc.edu \\ Sheng-He Huang, Department of Paediatrics, Saban Research Institute of Children's Hospital, University of Southern California, Los Angeles, California, United states, \\ Tel: +86 27 87792217; E-mail: yhzhou@hust.edu.cn
}

Received date: April 25, 2016; Accepted date: May 02, 2016; Published date: May 09, 2016

Copyright: (C) 2016 Huang SH, et al. This is an open-access article distributed under the terms of the Creative Commons Attribution License, which permits unrestricted use, distribution, and reproduction in any medium, provided the original author and source are credited.

\begin{abstract}
The vast majority of microbes form a healthy symbiotic 'superorganism' with the hosts. There are two types of symbiosis (Sym), exosymbiosis (e.g. microbiota) and endosymbiosis (e.g. mitochondria). It has been suggested that the exo-endo Sym balance (EESB) highly contribute to maintain the host homeostasis. However, alterations to the EESB caused by microbial (e.g. bacterial and viral pathogens) and non-microbial factors (e.g. substance abuse, diet and/or lifestyle) can disturb this symbiotic relationship and promote disease, such as inflammatory bowel diseases and acquired immune deficiency syndrome (AIDS). Progressive AIDS caused by human immunodeficiency virus (HIV) and simian immunodeficiency virus (SIV) is characterized by systemic inflammation, opportunistic infection and malignant disorders resulting from generalized immune activation-mediated destruction of the healthy symbiotic super organism. Two extreme phenotypes are present in both HIV and SIV infections, including slow or rapid progression to AIDS (Pat: pathogenesis) in a majority of the infected human subjects and the non-natural primate host (i.e. rhesus macaques, RMs), and nonprogression to AIDS (Sym) in a minority of the infected people and the natural primate hosts (i.e. sooty mangabeys, SMs). Recently, it has been demonstrated that both exosymbiotic and endosymbiotic disorders contribute to the development of AIDS through infectomic studies of the extreme phenotypes of HIV/SIV infections. The involvement of the EESB in the pathogenesis and therapeutics of HIV infections is becoming increasingly clear. Indeed, many changes in the microbial diversity, abundance and composition of the gut microbiota and mitochondrial functions have been reported in HIV/AIDS, suggesting that there exist EESB problems in this disease. HIV virotoxins have been implicated in exploiting mitochondria to promote the targeted progressive and inexorable depletion of key immune cells (e.g. CD4 T cells), a hallmark of HIVISIV infections. These findings support the notion that the exo-endo Sym imbalance (EESI) may play a central role in epidemiology, pathogenesis and management of infectious diseases, including AIDS caused by HIV-1 and SIV. Correction of the EESI problems in HIVISIV infections may lead to a rational control of AIDS.
\end{abstract}

Keywords: Exosymbiosis; Symbiosis; Endosymbiosis; Exo-Endo symbalance; Exo-Endo symimbalance; HIV; AIDS

\section{Introduction}

Infectious diseases, including HIV-1/AIDS, continue to be the leading cause of morbidity and mortality worldwide despite the availability of effective antimicrobial agents and vaccines over the last several decades [1].

The continual emergence of new pathogens, re-emergence of old pathogens, and the rising crisis of antibiotics resistance will certainly heighten the global impact of microbial threats to human health today. HIV/AIDS is the most salient emerging disease in modern medicine [2]. Progressive AIDS caused by HIV and SIV is characterized by systemic inflammation, opportunistic infection and malignant disorders resulting from generalized immune activation-mediated destruction of the host defence system [3]. Pathogenesis, prevention, treatment and cure of HIV-1 infection remain one of the greatest challenges in modern medicine since the discovery of this disease 1981 $[4,5]$. There are usually two basic phenotypes [pathogenesis (Pat) and symbiosis (Sym)] of microbial infection including human HIV/AIDS $[6,7]$. However, the conventional wisdom places emphasis on the pathogenic process-namely antagonism-rather than mutualism [1]. Two extreme phenotypes are present in both HIV-1 and SIV infections, including slow or rapid progression to AIDS (Pat) in a majority of the infected human subjects and the non-natural primate host (i.e., rhesus macaques, RMs), and nonprogression to AIDS (Sym) in a minority of the infected people and the natural primate hosts (i.e., sooty mangabeys, SMs) [7]. Infectomic insight into the differences in phenotypes of HIV-1/SIV infections has been essential for uncovering this relationship and conquering AIDS. The most fundamental issue in infectomics is how to dissect the dynamic Sym-Pat duality in microbial infections $[1,8]$. There are two types of symbiosis, exosymbiosis (e.g, microbiota) and endosymbiosis (e.g. mitochondria). It has been proposed that the Exo-Endo Sym (EES) balance is essential for health and that the EES imbalance (EESI) plays a central role in the pathogenesis of infectious diseases, including AIDS caused by HIV-1 and SIV [8]. This notion is further supported by our infectomic analysis of the two extreme phenotypes of SIV infection [7].

\section{Exo-Endo Sym Balance (EESB) and health}

There are two types of symbiotic relationships, including those associations in which one organism lives on another (exosymbiosis, 
e.g. microbiota living on the body surface of the host), and where one partner lives inside the cells or tissues of the other (endosymbiosis, such as mitochondria in eukaryotic cells) [8]. The vast majority of microbes form a healthy symbiotic 'superorganism' with the hosts. The ecology and evolution of the exosymbiotic relationships between microbes and their hosts are essential and fundamental for health. Most of our microbial commensals reside in our gastrointestinal (GI) track packed with up to 100 trillion $\left(10^{14}\right)$ microbes, with up to 1000 or so different bacterial species $[8,9]$. The GI tract microbiota constitutes a natural defensive barrier to infection. They may provide a number of positive health benefits for their hosts. These include stimulating the immune system, protecting the host from microbial invasion, aiding digestion, maintaining host homeostasis and modulating energy balance. The GI tract is a complex ecosystem formed by the symbiotic alliance of GI epithelium, immune cells, and microbiota. Just as most exosymbiotic microbes form a healthy symbiotic superorganism with the hosts, an elegant balance of this symbiotic relationship is essential to the development and function of immune system. A few recent studies showed that mitochondria, an endosymbiotic organelle, could play an important role in the cross-talk between microbiota and host [10-12]. There are many common features shared by both microbiota and mitochondria due to the prokaryotic origin of mitochondria. These include similar structures/functions, protein degradation pathways, sensitive to antibiotics and the presence of their DNAs in the nuclear genome [10]. Reactive oxygen species (ROS) produced by mitochondria may be important for the innate immune response and inflammation induced by microbial pathogens. Mitochondria may affect ROS signaling induced by the microbiota to regulate the gut epithelial barrier. It has been reported that health problems such as obesity, diabetes mellitus, Crohn's disease or even autism and depression are associated with specific microbiota diversity and quality that may be regulated by mitochondria $[10,11]$. These findings suggest that the exo-endo sym balance is essential for health.

\section{Exo-Endo Sym Imbalance (EESI) and AIDS caused by HIV-1 and SIV}

It is well known that both microbial translocation across the gut barrier (disturbing exosymbiosis) and mitochondria mediated disorders (dysregulation of endosymbiosis) are involved in the pathogenesis of AIDS [8]. HIV and SIV infections can contribute to increased bacterial translocation and chronic immune activation through altering the intestinal immune system and microbiota interplay [13-15]. Increased abundances of Proteobacteria and decreased abundances of Firmicutes are observed in colon biopsies of HIV-1-infected subjects compared with that in uninfected donors. There is a significant increase in Prevotella and decrease in Bacteroides in HIV-1-infected subjects. The increase in Prevotella abundance was correlated with increased numbers of activated colonic $\mathrm{T}$ cells and myeloid dendritic cells during HIV-1 infection. These findings suggest that an important relationship exists between altered exosymbiotic microbial communities and the mucosal immune system during chronic HIV-1 infection. Meanwhile, endosymbiotic (mitochondrial) disorders also contribute to the pathogenesis of HIV infection and HIV-associated neurocognitive disorders (HAND) [16-18]. Mitochondria can directly influence the progression of AIDS, including the viral infectivity, the course of HIV-1 infection, and the prevalence of side effects from the primary HIV-1 therapy, highly active antiretroviral therapy (HAART). Point mutations and deletion of mitochondrial DNA can lead to decreasing HIV-1 infection [16]. Mitochondria play a key role in the production of energy and the induction of cellular apoptosis, which can be dysregulated by HIV viral genes [18]. We have demonstrated recently that both exosymbiotic and endosymbiotic disorders contribute to the pathogenesis of AIDS through infectomic studies of the Sym/Pat phenotypes of HIV and SIV infections [6-8,19]. The Pat+ phenotype in SIV-infected RMs is specifically associated with disorders in the protein ubiquitin proteasome system, p53, granzyme A, gramzyme B, polo-like kinase, Glucocorticoid receptor, oxidative phosyphorylation and mitochondrial signaling. Mitochondrial (endosymbiotic) dysfunction is solely present in RMs. Sym+ pattern changes identified in SIVinfected SMs are the pathways contributing to the host defense against viral infection, including interferon signaling, BRCA1/DNA damage response, PKR/INF induction and LGALS8. The genes LGALS8 and IL-17RA, which positively regulate the barrier function of the gut mucosa and the immune homeostasis with the gut microbiota (exosymbiosis), were significantly differentially expressed in RMs and SMs. These findings suggest that the exo- and endo-sym imbalance (EESI) may play a central role in the pathogenesis of in HIV/SIV infections.

\section{Suspected involved mechanisms}

The underlying mechanisms contributing to the EESI in HIV/SIV infections remain unclear. Recently, the concept of ecoimmunity has been proposed to explore the interplay between the immune system and its ecology [8]. The immune system and microbial communities are viewed as two interrelated and dynamically co-evolving components of a predator-prey ecosystem. These efforts emphasize the interchange between the organism and its ecological environments with more holistic consideration of immune regulation through chemical and genetic communications. HIV/SIV infections can induce significant changes in the diversity, abundances and components of microbiota, which disturb ecological environments. HIV virotoxins have been implicated in exploiting mitochondria to promote the targeted progressive and inexorable depletion of key immune cells (e.g. CD4 T cells), a hallmark of HIV/SIV infections. It disturbs the ecoimminty and directly contributes to disease progression and immunodeficiency [18]. Correction of the ecoimmunity problems and EESI in HIV/SIV infections may lead to a rational control of AIDS.

\section{Conclusion}

The advent of microbiome sequencing and infectomic technologies has greatly improved the characterization of virotoxins and host factors that contribute to the pathogenesis and therapeutics of HIV/AIDS. However, a better understanding of the EESB in health subjects and EESI in patients with HIV-1 infections requires further structural and functional studies of both the exo and endo sym systems and the connectivity between the two systems. Most of the studies published in this regard have been carried out without characterizing the EESB/ EESI and their connection. Investigations should also consider the use of the ecoimmunity approaches to study the ecoimmunity system that is modulated both by external and internal factors in relation to molecular and cellular pathways involved in EESI associated with HIV infections. In summary, the role of EESI in HIV/SIV infections is increasingly evident and perhaps represents a new central approach towards the development of rational therapeutic interventions in AIDS.

\section{Funding Information}

This work was supported by grants from National Institutes of Health, National Institute of Neurological Disorders and Stroke and National Institute on Drug Abuse [NIH/NIDA 5R03DA034515, NIH/ NINDS 5R21NS083967]. 
Citation: Huang SH, Zhou YH (2016) Infectomic Insights into the Roles of Exosymbiosis-Endosymbiosis Imbalance (EESI) in HIV-1 and SIV Infections. J Data Mining Genomics \& Proteomics 7: 196. doi:10.4172/2153-0602.1000196

Page 3 of 3

\section{References}

1. Huang SH, Zhou W, Jong A (2008) Focal point theory models for dissecting dynamic duality problems of microbial infections. J Biomed Biotechnol.

2. Morens DM, Fauci AS (2013) Emerging infectious diseases: threats to human health and global stability. PLoS Pathog 9: e1003467.

3. d'Ettorre G, Paiardini M, Ceccarelli G, Silvestri G, Vullo V (2011) HIVassociated immune activation: from bench to bedside. AIDS Res Hum Retroviruses 27: 355-364.

4. Palmisano L, Vella S (2011) A brief history of antiretroviral therapy of HIV infection: success and challenges. Ann Ist Super Sanita 47: 44-48.

5. Caceres CF, Koechlin F, Goicochea P (2015) The promises and challenges of pre-exposure prophylaxis as part of the emerging paradigm of combination HIV prevention. J Int AIDS Soc.

6. Ma C, Zhou Y, Huang SH (2011) Inequalities and duality in gene coexpression networks of HIV-1 infection revealed by the combination of the double-connectivity approach and the Gini's method. J Biomed Biotechnol.

7. Yang ZW, Jiang YH, Ma C, Silvestri G, Bosinger SE, et al. (2016) Coexpression Network Analysis of Benign and Malignant Phenotypes of SIV-infected Sooty Mangabey and Rhesus Macaque. PLoS One.

8. Huang SH, Tang SQ, Bo T, Xia HW, Jong A (2013) Ecological infectomics (Ecoinfectomics): symbiotic solutions to microbial infections, AntiInfective Agents 11: 93-99.

9. Rajilić-Stojanović M, de Vos WM (2014) The first 1000 cultured species of the human gastrointestinal microbiota. FEMS Microbiol Rev 38: 996-1047.

10. Saint-Georges-Chaumet Y, Edeas M (2016) Microbiota-mitochondria inter-talk: consequence for microbiota-host interaction. Pathog Dis 74 ftv096.
11. MacFabe DF (2015) Enteric short-chain fatty acids: microbial messengers of metabolism, mitochondria, and mind: implications in autism spectrum disorders. Microb Ecol Health Dis 26.

12. Zorov DB, Plotnikov EY, Silachev DN, Zorova LD, Pevzner IB, et al. (2014) Microbiota and mitobiota. Putting an equal sign between mitochondria and bacteria. Biochemistry (Mosc) 79: 1017-1031.

13. Dillon SM, Lee EJ, Kotter CV, Austin GL, Dong Z, et al. (2014) An altered intestinal mucosal microbiome in HIV-1 infection is associated with mucosal and systemic immune activation and endotoxemia. Mucosal Immunol 7: 983-994.

14. Moeller AH, Shilts M, Li Y, Rudicell RS, Lonsdorf EV, et al. (2013) SIVinduced instability of the chimpanzee gut microbiome. Cell Host Microbe 14: 340-345.

15. Noguera-Julian M, Rocafort M, Guillen Y, Rivera J, Casadella M, et al. (2016) Gut Microbiota Linked to Sexual Preference and HIV Infection. EBioMedicine 5: 135-146.

16. Villeneuve LM, Purnell PR, Stauch KL, Callen SE, Buch SJ, et al. (2016) HIV-1 transgenic rats display mitochondrial abnormalities consistent with abnormal energy generation and distribution. J Neurovirol.

17. Vartak-Sharma N, Nooka S, Ghorpade A (2016) Astrocyte Elevated Gene-1 (AEG-1) and the A(E)Ging HIV/AIDS-HAND. Prog Neurobiol .

18. Shedlock DJ, Hwang D, Choo AY, Chung CW, Muthumani K, et al. (2008) HIV-1 viral genes and mitochondrial apoptosis. Apoptosis 13: 1088-1099.

19. Ma C, Huang SH, Zhou YH (2014) Measuring Inequalities in Gene Coexpression Networks of HIV-1 Infection Using the Lorenz Curve and Gini Coefficient. J Data Mining Genomics Proteomics 5: 1-4. 